Pablo Galindo-Moreno

Peter Nilsson

Paul King

Jonas Becktor

Stefano Speroni

Alexander Schramm

Carlo Maiorana

\title{
Clinical and radiographic evaluation of early loaded narrow diameter implants - 1-year follow-up
}

Key words: implant, incisor, marginal bone loss, narrow, small diameter

Authors' affiliations:

Pablo Galindo-Moreno, Oral Surgery and Implant

Dentistry Department, School of Dentistry,

University of Granada, Granada, Spain and

Department of Periodontics and Oral Medicine,

School of Dentistry, University of Michigan Ann

Arbor, MI, USA

Peter Nilsson, Department of Oral \& Maxillofacial

Surgery, The Institute for Postgraduate Education, Jönköping, Sweden

Paul King, Restorative Dentistry, University of Bristol Dental Hospital \& School, Bristol, UK

Jonas Becktor, Department of Oral \& Maxillofacial

Surgery, University Hospital (Rigshospitalet), Copenhagen, Denmark

Stefano Speroni, Department of Implantology

Fondazione IRCSS Ca' Granda Ospedale Maggiore

Policlinico Milano, University of Milan, Milan,

Italy

Alexander Schramm, Department of Oral and

Maxillofacial Surgery, German Federal Armed

Forces Hospital, Ulm, Germany

Carlo Maiorana, Oral Surgery and Implantology

Fondazione IRCSS Ca' Granda Ospedale Maggiore

Policlinico Milano, University of Milan, Milan,

Italy

\section{Corresponding author:}

Dr Pablo Galindo-Moreno

C/Recogidas, $395^{\circ}$ Izq.

18005 Granada

Spain

Tel.: +34958520658

Fax: +34958 520658

e-mail: pgalindo@ugr.es and pablogm@umich.edu

Date:

Accepted 23 May 2011

To cite this article:

Galindo-Moreno P, Nilsson P, King P, Becktor J, Speroni S,

Schramm A, Maiorana C. Clinical and radiographic

evaluation of early loaded narrow diameter implants - 1-year

follow-up.

Clin. Oral Impl. Res. 23, 2012, 609-616

doi: $10.1111 / j .1600-0501.2011 .02254 . \mathrm{x}$

\section{Abstract}

Objective: To evaluate the clinical performance of Astra Tech OsseoSpeed ${ }^{\mathrm{TM}}$ TX $3.0 \mathrm{~S}$ implants using one-stage surgical procedure and early loading in the anterior region.

Material and methods: This is a prospective, single arm, multi-centre study. Patients missing teeth at positions 12, 22 and 32-42 were eligible to enter the study. The implants (OsseoSpeed ${ }^{\mathrm{TM}}$ TX 3.0S) used in the study were of $3 \mathrm{~mm}$ diameter and of different lengths. One-stage surgery was performed, and healing abutments were used during the 6-10 weeks healing period. Clinical and radiographic examinations were assessed at implant installation, loading and at the 6- and 12-month follow-up visits.

Results: Ninety-seven implants were placed in 69 patients at six different study centres in Denmark, Germany, Italy, Spain, Sweden and the United Kingdom. The survival rate was $95.9 \%$. No implants have been lost after loading (100\% survival rate after loading). Mean marginal bone loss 1 year after installation was $0.065 \mathrm{~mm}(\mathrm{SD}=1.018)$. The frequency of bone loss $\geq 1 \mathrm{~mm}$ was $6.6 \%$ and $51.3 \%$ of the implants demonstrated no bone loss or even bone gain from the surgical visit to the first year follow-up visit. Mean probing pocket depth and gingival zenith score were stable from crown placement to the 6- and 1-year follow-up visits.

Conclusion: Treatment with OsseoSpeed ${ }^{\mathrm{TM}}$ TX $3.0 \mathrm{~S}$ implants is a safe and predictable option in the anterior region where physical space is limited. Minimal marginal bone loss was observed during the first year follow-up.

Some clinical rehabilitation scenarios represent a challenge for clinicians. This is often the case where missing teeth need to be replaced in the anterior region of the mouth. Dental implants in this region are in some cases recommended instead of orthodontic space closure, autotransplantation or conventional prosthetic replacement (Thilander et al. 1999). However, replacing upper lateral incisors or lower central incisors with standard diameter implants can constitute a challenge, due to the anatomical features of these teeth or the space available. A reduced buccolingual dimension may not allow the placement of a standard diameter implant without the risk of implant thread exposure (Romeo et al. 2006), and due to the narrow mesio-distal width, a hazard to the neighbouring teeth or altering the interproximal bone (Cardaropoli et al. 2006). The placement of an implant closer than $1.5 \mathrm{~mm}$ to the adjacent tooth may result in loss of proximal bone height during healing (Tarnow et al. 2000).

Aesthetic considerations are critical in this area, and will be influenced by implant placement. The bone level constitutes the base for the supracrestal soft tissue around the implant, conditioning the final position of the papillae and the harmony between the new restoration, the remaining teeth and the surrounding soft tissue. Classically, soft tissue topography is determined by parameters such as contact point position (Tarnow et al. 1992), crown dimensions and temporization (Jemt 1999), tooth-implant distances (Froum et al. 2007) and implant diameter (Chang et al. 1999).

In this sense, narrow diameter implants represent an alternative treatment option in areas with limited space. Some clinical stud- 
ies have reported higher success rates for standard diameter implants in comparison with narrow diameter implants in soft quality bone (van Steenberghe et al. 1990). An increasing risk of implant fracture following loading has been reported for narrow implants (Schwarz 2000). There are, however, studies that have focused on the use of small diameter implants that have documented positive outcomes (Zinsli et al. 2004; Romeo et al. 2006; Degidi et al. 2008).

Narrow implant indications include clinical situations with reduced amount of interradicular bone, narrow ridges or reduced mesio-distal prosthetic space (Davarpanah et al. 2000), which are often found in clinical situations such as congenitally missing incisors, retained primary incisors that are lost, missing mandibular incisors, space collapse in anterior area with a lack of orthodontic therapy or reduced interdental space after orthodontic movements (Froum et al. 2007).

The definition of a narrow diameter implant is still controversial, and depends on the specific manufacturer or author. In general terms, a narrow implant has a diameter $<3.4 \mathrm{~mm}$ (Quek et al. 2006), although it is important to distinguish between narrow implant and mini-implant, which normally constitutes a one piece implant, with a diameter $<3 \mathrm{~mm}$ (Jofre et al. 2010) and with an orthopaedic bone screw form. Normally these implants are used for orthodontic anchorage (Lee et al. 2010) or have been considered as transitional when they are used to retain prostheses (Ohkubo et al. 2006). An implant can be considered to be definitive if the bone around it remains stable after receiving a physiological load. Important mini-implants limitations are loosening, deformation and fracture during treatment and at removal because their diameters are quite small (Kanie et al. 2004). Equally, usefulness of small diameter implants has to be discussed with an awareness of their potential limitations. It has been estimated that a $3.3 \mathrm{~mm}$ diameter, screw-shaped, commercially pure titanium implant possesses $25 \%$ less resistance to fracture when compared with a similar regular (3.5-4 mm) diameter implant. Thus, decreasing the diameter also means increasing the risk for implant fracture because of reduced mechanical stability and increasing the risk for overload (Olate et al. 2010).

For implants with conventional diameters, the average bone loss in the first year at the proximal sites is $1.6 \mathrm{~mm}$ (Cardaropoli et al. 2006). Some authors consider successful treatment is achieved if the marginal bone loss is $<2 \mathrm{~mm}$ (Misch et al. 2008). These arguments, however, have not been evaluated for small diameter implants. It has been reported that increasing implant diameter resulted in as much as a 3.5 -fold reduction in crestal strain (Petrie \& Williams 2005). The influence of the implant diameter on crestal bone strains dominates over the effect of the implant's length or taper (Petrie \& Williams 2005), therefore, an implant with a larger diameter helps to reduce the maximum stress/strain values between the bone and the implant (Qian et al. 2009). From this point of view, the marginal bone loss could increase when using narrow implants, and even more so in the anterior area.

The aim of this analysis therefore was to evaluate the marginal bone loss and the final outcome of single tooth replacement in the anterior region with limited physical space by the use of OsseoSpeed ${ }^{\mathrm{TM}}$ TX 3.0S implants (Astra Tech AB, Mölndal, Sweden).

\section{Material and methods}

This study was designed as a prospective, single arm, international multicentre study. Patients in need of single tooth replacement in positions 12, 22 and 32-42 (FDI) were recruited at six different study sites in Denmark, Germany, Italy, Spain, Sweden and the United Kingdom where approval from each local ethics committee was obtained. This study has been registered in the http://www. clinicaltrials.gov, from the US National Institutes of Health, identified as NCT00646113, from March 25, 2008.

All patients received detailed oral and written information on the study and signed a written consent before the start of the treatment. The recruited subjects had to fulfil the following criteria: good general health, smoking $\leq 10$ cigarettes per day, absence of oral and dental disorders, single tooth loss with neighbouring teeth in normal occlusion, recipient sites for implants that had healed for 2 months following tooth extraction (immediate implant placement was allowed after extracting a primary tooth). Only one study implant per patient was installed, except for patients missing both contra laterals, where both positions were allowed to be treated with study implants.

A total of 72 patients consented to the study. Three patients were excluded from the study, one patient was under 18 years of age, one patient was missing the canines and one patient did not turn up for installation of the study implant. In this analysis 69 patients with 97 implants have been included. A total of 36 men and 33 women, mean age 32 years $(\mathrm{SD}=15)$ participated in the study.

The implants used in the current study were OsseoSpeed ${ }^{\mathrm{TM}}$ TX $3.0 \mathrm{~S} 3 \mathrm{~mm}$ diameter with lengths of 11,13 and $15 \mathrm{~mm}$. The surgeon determined implant length from case to case.

\section{Surgical procedure}

All patients were treated with antibiotic prophylaxis $(2 \mathrm{~g}$ Amoxicillin or $600 \mathrm{mg}$ Clindamycin) $1 \mathrm{~h}$ before surgery. After local anaesthesia, incisions were made at the neighbouring teeth and connected by a crestal incision over the edentulous area. Flaps were elevated to expose the bone ridge. The implants were installed according to the methods described in the implant system manual for surgical procedures (Astra Tech AB, Mölndal, Sweden). Each surgeon determined the final drill (diameter of 2.7 or $2.85 \mathrm{~mm}$ ) to prepare the implant bed depending on the bone quality. The OsseoSpeed ${ }^{\mathrm{TM}}$ TX 3.0S has a tapered apex in to facilitate implant insertion, even when the final drill diameter is $2.7 \mathrm{~mm}$, which sometimes was required to get primary stability of the implant. The use of an osteotome technique was not permitted. Only grafting with autogenous bone chips harvested during the surgery was allowed around the implant site. After implant installation the flaps were sutured around a trans-mucosal healing abutment. The patients were given full post-operative instructions and recommended to rinse with chlorohexidine twice daily for 10 days after surgery.

\section{Prosthetic procedure}

If desired by the patient a temporary prosthesis not connected to the implant could be placed after the surgery. The sutures were removed after 7-13 days and impression was taken 5-7 weeks after implant installation. 6-10 weeks after implant installation the permanent crown restoration was cemented on an individually modified standard titanium transmucosal abutment (TiDesign ${ }^{\mathrm{TM}}$; Astra Tech AB). The abutments were installed according to the methods described in the implant system manual for surgical procedures and cement retained restorations (Astra Tech $A B)$. A study flow chart is presented in the Fig. 1. Metal-ceramic cemented-retained crowns were positioned in all the cases, over a $3 \mathrm{~mm}$ specific TiDesign ${ }^{\mathrm{TM}}$ abutment, that could be straight or $15^{\circ}$ angulated, depending of the clinician criteria. In some specific cases, those that suffered fracture of the abutment, a customized abutment (Atlantis $^{\text {TM}}$; 


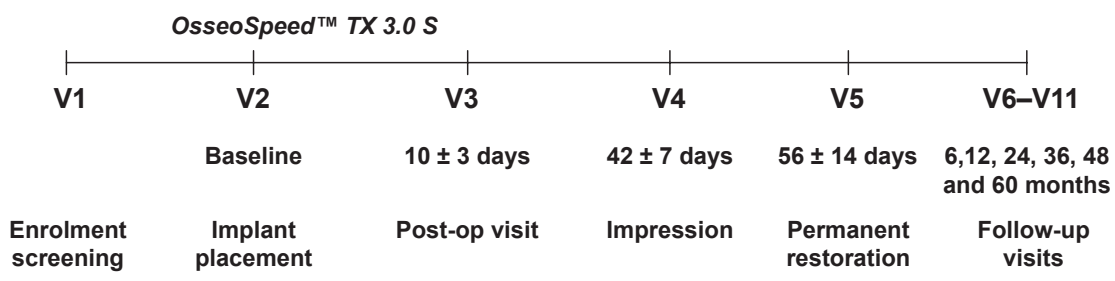

Fig. 1. Study flow chart.

Astra Tech $\mathrm{AB}$ ) was supplied for crown reposition.

\section{Clinical and radiological examinations}

Clinical and radiological examinations were conducted at implant installation, placement of the crown, and at 6- and 12-month followup visits. Intraoral radiographs were taken with a parallel technique using the study sites equipment. An independent experienced radiologist analysed all intraoral radiographs. The marginal bone levels were determined as the distance from the mesial and distal interproximal bone to the reference point (the junction between the machined bevel and the micro threads) and presented as a mean of the two values. The condition of the periimplant mucosa was measured by probing pocket depth (PPD) and bleeding on probing on four surfaces of each implant restoration. Maintenance of the soft tissue in the aesthetic zone was followed by measuring the gingival zenith, i.e. the most apical aspect of the buccal gingiva to the incisal edge of the crown.

\section{Statistical analysis}

For the statistical analysis the patients were divided into groups, based on criteria such as bone quality, bone quantity, smoking history, healing time, drilling protocol, implant length and grafting. When testing, if the expected value of a parameter was equal between the groups, the Student's $t$-test was used. No adjustment for multiple comparisons was made. Microsoft Office Excel 2003 was used for the statistical analyses. All tests were two-sided and a $P$-value below $5 \%$ discussed as statistically significant even if no adjustment for multiple comparisons were made.

\section{Results}

A total of 72 patients consented to participate in the study. Data for three patients have not been analysed, two of these patients were excluded since they did not fulfil all eligibility criteria and the third patient did not come for the installation of the implant. In this analysis 69 patients (97 implants) were followed, 36 men and 33 women between 18 and 72 years of age. The reason for the edentulism in the study area in our patients 197 spaces) was agenesis in $62(63.9 \%)$ cases, endodontic disease in $14(14.4 \%)$ locations, periodontal disease in $11(11.3 \%)$ areas, root fracture in $8(8.2 \%)$ tooth, non-restorable caries in 1 case, and unknown in 1 case. Eleven patients $(15.9 \%)$ were smokers, and two $(2.9 \%)$ of these were occasional smokers. Ten patients $(14.5 \%)$ reported previous history of periodontitis, and $10.1 \%$ of the patients showed bruxism habits. All patients received one or two OsseoSpeed ${ }^{\mathrm{TM}}$ TX 3.0S implants in positions 12, 22 and 32-42 (FDI). In total, 45 implants length $11 \mathrm{~mm}, 39$ implants length $13 \mathrm{~mm}$ and 13 implants length $15 \mathrm{~mm}$, were placed in the study (Fig. 2). The bone quality and quantity at implant sites is highlighted in Table 1, according to Lekholm and Zarb's (1985) classification.

Four implants have been lost at three different clinics. Three of these implants were lost because of insufficient healing and one implant was lost due to infection. One of the patients that lost one implant withdrew the consent to participate in the study despite having a second study implant. All four implants were lost during the healing period, before loading of the implant $195.9 \%$ total survival rate). No further implant loss has occurred between loading and the 1-year follow-up visit $(100 \%$ survival rate after loading).
Other complications were three fractured abutments (TiDesign ${ }^{\mathrm{TM}}$ ) in two different patients. Two of the fractured abutments were in the same patient due to offset buccolingual placement of the implants that created a lever and increased bending forces on the abutments. One of the cases was solved using a customized patient specific abutment (Atlantis ${ }^{\mathrm{TM}}$ ). The third broken abutment was after clinical inspection, determined to relate to excessive occlusal loading. In addition four crowns had to be recemented during the first year in function.

The mean marginal bone loss from surgery to loading was $0.439 \mathrm{~mm} \quad$ ( $\mathrm{SD}=0.893$ ), 6 months after surgery bone loss was $0.315 \mathrm{~mm}(\mathrm{SD}=0.925)$ and bone loss 1 year after surgery was $0.065 \mathrm{~mm}(\mathrm{SD}=1.017)$ on implant level (Fig. 3). The frequency of implants experiencing bone loss $\geq 1 \mathrm{~mm}$ was $6.6 \%$ and $51.3 \%$ of the implants demonstrated no bone loss (or even bone gain) between implant installation and the first year follow-up visit (Fig. 4). No relationship

Table 1. Distribution in the matrix bone quality/ bone quantity

\begin{tabular}{lrrrrrr}
\hline \multirow{7}{*}{$\begin{array}{l}\text { Bone } \\
\text { quality }\end{array}$} & $\mathrm{A}$ & $\mathrm{B}$ & $\mathrm{C}$ & $\mathrm{D}$ & $\mathrm{E}$ & Total \\
\cline { 2 - 5 } 1 & 1 & & & & & 1 \\
2 & 20 & $28(3)$ & 13 & & 61 \\
3 & 9 & $22(1)$ & 1 & & 32 \\
4 & & 3 & & & & 3 \\
Total & 30 & 53 & 14 & 0 & 0 & 97 \\
\hline \multicolumn{4}{l}{ Lost implants within parenthesis. } \\
\hline
\end{tabular}




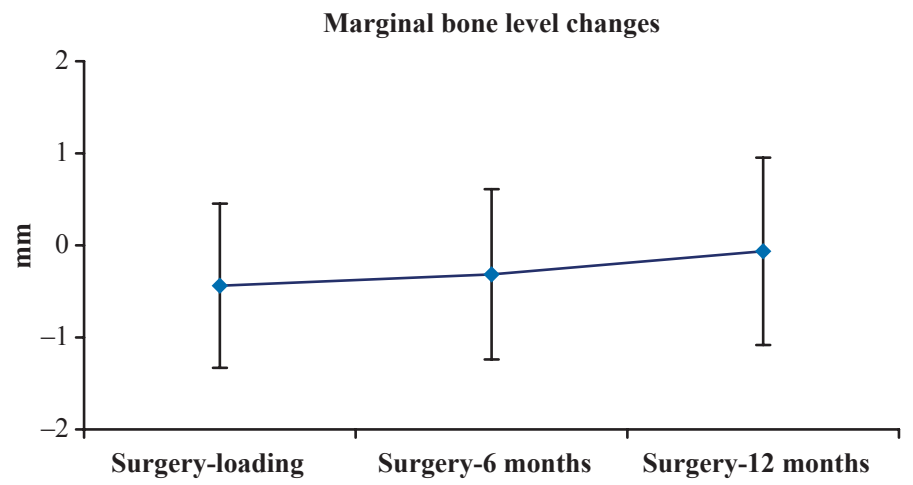

Fig. 3. Marginal bone loss level evolution from surgery to 1-year follow-up visit (visits 2-7).

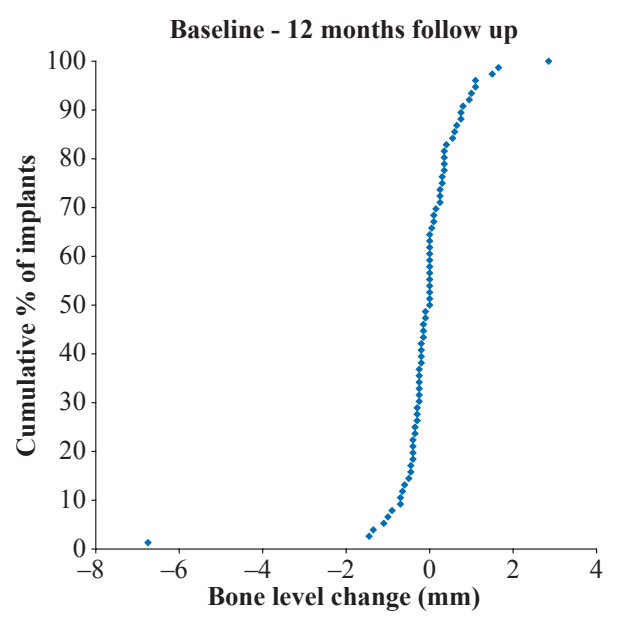

Fig. 4. Bone level change per implant.

was found between origin of edentulism and marginal bone loss. The marginal bone loss was not affected by the nicotine use in any of its modalities, implant length, bone grafting prior to or during the implant surgery, or bone quality. Statistical differences in marginal bone loss, however, were observed regarding bone quantity. Bone quantity $\mathrm{B}$ showing an average marginal bone loss of $0.11 \mathrm{~mm}$ compared to 0.31 and $0.74 \mathrm{~mm}$ bone loss for bone quantity A and $\mathrm{C}$, respectively $(P=0.295$ and 0.015 , respectively, Student's $t$-test). Last drill showed a tendency to affect the marginal bone loss after 1 year compared to loading. Both 2.7- and $2.85 \mathrm{~mm}$ diameter drills were used as last drill. Using a wider $2.85 \mathrm{~mm}$ last drill demonstrated $0.25 \mathrm{~mm}$ average bone gain 1 year after implant installation compared to the crown placement visit. Whilst using a $2.7 \mathrm{~mm}$ last drill resulted in a $0.39 \mathrm{~mm}$ average bone loss, in the same period. This difference was statistically significant $(P=0.0068$, Student's $t$-test). This bone level difference, however, was not present at the 6-month follow-up visit. Regarding the healing time from surgery to loading, the shorter this period was, the higher marginal bone loss was observed from placement of the crown and the 6- and 12-month follow-up visits. In this analysis we compared healing time periods $\leq 55$ and $\geq 56$ days. For the 6-month followup visit the marginal bone loss was 0.49 and $0.06 \mathrm{~mm}$, respectively $(P=0.0635$, Student's $t$-test). This significance was not confirmed at the 1-year follow-up visit where the marginal bone loss was 0.51 and $0.27 \mathrm{~mm}$, respectively $(P=0.4027$, Student's $t$-test $)$. The average healing period was 70.25 $(\mathrm{SD}=38.86)$ days.

The mean PPD at crown placement was $1.96 \mathrm{~mm}(\mathrm{SD}=0.714)$ although the pocket depth tended to decrease after 6 months to $1.83 \mathrm{~mm}(\mathrm{SD}=0.647)$, and then remaining stable with $1.82 \mathrm{~mm} \quad(\mathrm{SD}=0.914)$ after 1 year. Moreover, bleeding on probing around the implants at crown placement was observed for $33.3 \%$ of the implants, at the 6 month follow-up visit bleeding was observed for $46.5 \%$ of the implants, and at the 1 -year follow-up visit bleeding was observed for $34.1 \%$ of the implants.

The gingival zenith score was stable from crown placement to the 6-month and 1-year follow-up visits and thereby indicating well maintained soft tissue around the implants. The mean gingival zenith score was $8.95 \mathrm{~mm}$ $(\mathrm{SD}=1.764)$ at the definitive crown placement, $8.72 \mathrm{~mm}(\mathrm{SD}=1.859)$ after 6 months, and $8.66 \mathrm{~mm}(\mathrm{SD}=1.694)$ at the 1-year follow-up visit.

\section{Discussion}

Initially, narrow diameter implants were developed to allow for tooth restoration without the need for bone augmentation or orthodontic tooth movement in critical clinical scenarios. Narrow diameter implants were designed for specific clinical situations, such as placement of implants where bone width is narrow or where prosthetic space between adjacent teeth is limited. Replacement of lateral maxillary and mandibular incisors also represents an ideal indication for a narrow diameter implant type. The OsseoSpeed ${ }^{\mathrm{TM}}$ TX 3.0S implant has a tapered apex to facilitate implant insertion, even in under-prepared implant sites. The possibility of minimizing the diameter of the osteotomy might also be beneficial for the vascularization and thereby the osseointegration process. Another advantage with this implant is the enabling of implant placement when the interradicular space is limited.

Recently, several studies are available in the literature describing the success rate of narrow implants, but the terminology is quite confusing regarding how to classify an implant according to its diameter. Quek et al. (2006) classified implants as small or narrow $(3-3.4 \mathrm{~mm})$ regular $(3.75-4 \mathrm{~mm})$ and wide $(5-6 \mathrm{~mm})$. Mini-implant would represent any implant with a diameter smaller than $2.9 \mathrm{~mm}$. Distinguishing between them is crucial to accurately evaluate success rates (Avila et al. 2007).

Traditionally, narrow implants have shown lower survival rates than standard diameter implants. Albrektsson et al. (2007) evaluated 550 NobelDirect implants of different size, and showed that $20 \%$ of the $3 \mathrm{~mm}$ narrow diameter implants failed. However, other studies have reported better results for narrow diameter implants. Saadoun and Le Gall (1996) reported $89 \%$ survival rates after 8 -year follow-up in a large series of $3.25 \mathrm{~mm}$ implant diameter. Similar to that report, Andersen showed that the survival rate of $3.25-\mathrm{mm}$ self-tapping titanium implants $(93.8 \%)$ was lower than that of $3.75-\mathrm{mm}$ implants $(100 \%)$ over a 3 -year observation period (Andersen et al. 2001). Simultaneously, another clinical research reported an increased failure rates for implants $3-4 \mathrm{~mm}$ diameter in comparison with implants over $4 \mathrm{~mm}$ diameter, $7.3-2.7 \%$, respectively, after 3-year follow-up (Winkler et al. 2000). This trend has been constant over time, and similar findings have been reported by other authors, as Romeo et al. (2006) who reported survival rates about $92 \%$ and $97.7 \%$, for 3.3 vs. $4.1 \mathrm{~mm}$ diameter Straumman implants, respectively, after 7-year follow-up, or Renouard and Nisand (2006), who reported a 93.3$95.3 \%$ success rates to $3 \mathrm{~mm}$ implants in comparison with a $96-99.4 \%$ to $3.3 \mathrm{~mm}$ implants.

Some authors, conversely, have reported similar or higher survival rates for narrow 
diameter implants compared to conventional diameter implants. Survival rate for 3-3.4 mm diameter implants have been reported to be $100 \%$ (Cordaro et al. 2006), 99.4\% (Hallman 2001), 99.4\% (Degidi et al. 2008), 99\% (Block \& Kent 1993) or $98.7 \%$ (Zinsli et al. 2004), after different follow-up times from 1 to 8 years, with different type of implants and protocols. The present results are in accordance with most of these studies, showing $95.9 \%$ total survival rate before implant loading (Vigolo \& Givani 2000; Vigolo et al. 2004; Zarone et al. 2006; Reddy et al. 2008). In our study, however, no implants have been lost after loading (100\% survival rate after loading).

The current protocol introduces some differential points compared to other studies. Few two-piece implant systems with a diameter of $3 \mathrm{~mm}$ are available on the market today. Conceptually these implants could raise some negative concerns, such as an increased implant body fracture possibility, increased implant failure or prosthetic complications, such as screw getting loosened or fracture of the prosthetic abutment (Allum et al. 2008; Arisan et al. 2010). Noteworthy, to date no implant fractures have occurred in the present study. Moreover, the two-piece design of the OsseoSpeed ${ }^{\mathrm{TM}} \mathrm{TX} 3.0$ S provides added flexibility for the clinician by supporting a one or two stage surgical approach. Based on individual case requirements, this two-piece concept is also ideal for either conventional temporization techniques or, in other instances, immediate temporization for optimal restorative versatility (Martin 2010).

The study protocol introduced occlusal loading between the 6th and the 10th healing week. The OsseoSpeed ${ }^{\mathrm{TM}}$ TX $3.0 \mathrm{~S} \mathrm{~mm}$ implant has an internal connection, which implies higher force application all over the implant during the abutment screw and unscrew. According to the protocol, impressions were taken from the fifth to the seventh week after surgery, and this is in close relation to the early bone maturation. Current knowledge regarding alveolar bone healing processes indicates the possibility of osseointegration disruption (Cardaropoli et al. 2003; Araujo et al. 2005). We assume that three of our implant failures occurred due to incomplete bone healing at the time of impression taking. Longer healing period could potentially result in higher survival rates. One adverse event was reported in a patient with a history of smoking and periodontal disease. The implant rotated during the impression phase, and it was explanted and implanted again in the same surgical bed, and after eight additional weeks of healing this implant was stable and functional after loading during the first follow-up year, without any sign of infection or marginal bone loss.

Marginal bone loss represents an important indicator for peri-implant health. From success criteria established by Zarb in the early 1980s to the recent Pisa Consensus (Misch et al. 2008), a marginal bone loss around the implants up to $2 \mathrm{~mm}$ in the first year after placing the fixture has been accepted (Albrektsson et al. 1986). Some authors advocate that initial bone loss around the implant could be due to the formation of the so-called biological width (Bengazi et al. 1996; Cardaropoli et al. 2003).

Considering the available literature, some experimental studies have highlighted the possible implant diameter impact on the cortical bone around the implant neck. Cehreli and Akça showed that the use of narrow diameter implants as terminal support for three-unit fixed partial dentures resulted in an increase in stress and strain magnitudes around supporting implants in comparison with support from two standard solid screw implants (Cehreli et al. 2006). Using threedimensional finite element models it has been shown that increased implant diameter resulted in as much as a 3.5-fold reduction in crestal strain (Petrie \& Williams 2005). Some clinical findings have supported these concepts, establishing that length and diameter seemed to influence the marginal bone loss, with less bone loss for wider and longer implants (Degidi et al. 2008). However, in an extensive literature review it was established that no relationship existed between marginal bone loss and implant diameter (Renouard \& Nisand 2006). This affirmation was in accordance with the findings by Romeo et al. (2006), who did not find peri-implant bone resorption influenced by the implant diameter.

Reddy et al. (2008), reported a marginal bone loss of $2.33 \pm 0.73 \mathrm{~mm}$ at the time of restoration, $1.75 \pm 0.78 \mathrm{~mm} 6$ months after restoration, and a successive reduction of bone loss to $1.63 \pm 0.81 \mathrm{~mm}$ after 1 year in function for a one piece $3 \mathrm{~mm}$ implant. However, Polizzi and co-workers reported a marginal bone loss $<1 \mathrm{~mm}$ during the first year of function. This study was conducted on a two-piece $3-\mathrm{mm}$ implant system for 22 implants (Polizzi et al. 1999). Comfort et al. (2005) reported even less marginal bone loss, $0.41 \pm 0.17 \mathrm{~mm}$, using a $3.3 \mathrm{~mm}$ implant.

One of the primary aims of this analysis was to evaluate if the narrow implant diameter influenced the marginal bone loss for this newly developed two-piece 3-mm diameter implant system. In the present study the mean marginal bone loss from surgery to the definitive crown delivery was $0.44 \mathrm{~mm}$ ( $\mathrm{SD}=0.89$ ). But, similar to findings in other studies, a recovery of marginal bone was seen from loading to 1 year after surgery. In this study almost all the marginal bone lost from surgery to loading was recovered, a $0.065 \mathrm{~mm}(\mathrm{SD}=1.02)$ average bone loss from surgery to the 12-month follow-up was reported.

Some clinical variables seemed to affect the marginal bone in the study population. Marginal bone loss showed statistical differences regarding bone quality, with less marginal bone loss in the type 2 bone (Fig. 5). These findings could be explained by adapted and atraumatic preparation techniques, as well as the careful patient selection in terms of biomechanical conditions and bone density. The osseous quality or quantity identified at the time of surgery could therefore potentially promote a change in surgical technique. In this study the diameter of the last drill used to prepare the implant bed statistically affected the marginal bone loss. Less marginal bone loss was observed after 1 year when a wider final drill was used 0.25 vs. $-0.39 \mathrm{~mm} ;>2.7$ vs. $\leq 2.7 \mathrm{~mm}$ diameter drill, respectively, $P=0.0068$ ), indicating that a wider osteotomy is more favourable for maintaining the surrounding bone. Implant stability has been argued as an important factor to achieve final osseointegration, however, to introduce implants with a high installation torque could potentially result in bone resorption due to compromising bone vascularization as a result of increased strain on the bone walls of the osteotomy. Degidi et al. $(2007,2008)$ did not find any significant difference associated with bone quality (maxilla or mandible) when evaluating survival of narrow or wide diameter implants. However, they found a better outcome with regard to reduced crestal bone loss over time for shorter or narrower implants (Degidi et al. 2007).

An interesting finding in the present study is that a longer healing period before crown placement contributed to statistically significant less marginal bone loss between loading and the 6-month and the 1-year follow-up visits. This finding, together with a stable PPD and a stable crown to gingiva distance over time, using a one-stage surgical protocol can contribute to maintaining the integrity of the soft tissue around the implant. It is known that the reduction in the soft tissue thickness is accompanied by a significant, 
MBL change from visit 2

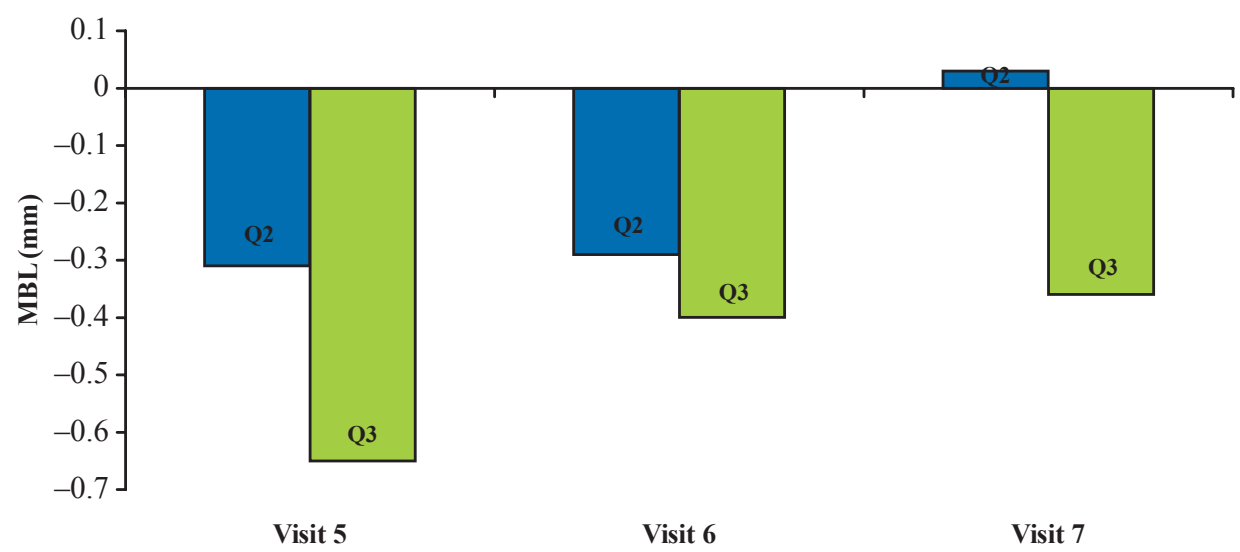

Fig. 5. Marginal bone level (MBL) changes from visit 2 (surgery) to visit 7 (1-year follow-up) depending on the bone quality.

apical displacement of the soft tissue margin around the definitive crown (Cardaropoli et al. 2006). Soft tissue integrity plays a principal role in the maintenance of the periimplant bone levels. In our study, absence of bleeding on probing at the 6-month visit was associated with the maintenance of marginal bone levels from the crown placement, $0.03 \mathrm{~mm}$ of bone gain vs. $0.31 \mathrm{~mm}$ of bone loss $(P=0.029$, Student $t$-test $)$ concerning those implants that showed bleeding on probing at the follow-up visit. Absence of inflammation could justify this difference, because the absence of pro-inflammatory cytokines protects this environment from protein-mediated resorption (Loomer et al. 1995; Kinane \& Lappin 2002).

In the present study, contrary to other previous studies, the marginal bone loss was not affected by the nicotine use, in any of the modalities (Galindo-Moreno et al. 2005), implant length (Degidi et al. 2008), grafting during the surgery or previous need of grafting or bone quality (Renouard \& Nisand 2006; Romeo et al. 2006). The differences regarding previous well-documented findings of a negative relationship between smoking and marginal bone maintenance could be because heavy smokers (over 10 cigarettes per day) were excluded in the study. Furthermore, no statistical relationship between

\section{References}

Albrektsson, T., Gottlow, J., Meirelles, L., Ostman, P.O., Rocci, A. \& Sennerby, L. (2007) Survival of NobelDirect implants: an analysis of 550 consecutively placed implants at 18 different clinical centers. Clinical Implant Dentistry $\oplus$ Related Research 9: 65-70.

Albrektsson, T., Zarb, G., Worthington, P. \& Eriksson, A.R. (1986) The long-term efficacy of currently used dental implants: a review and proposed implant length and marginal bone loss was found, although some studies have argued that the use of a longer implant resulted in less marginal bone loss around it (Petrie \& Williams 2005; Degidi et al. 2008). Other authors have, in accordance with our findings, highlighted this lack of influence between implant length and marginal bone loss (Renouard \& Nisand 2006), arguing that the stress forces are around the cortical bone, just over the first threads of the implants (Matsushita et al. 1990; Anitua et al. 2010). Moreover, no differences were found between implants placed in pristine bone in comparison with implants placed in grafted areas, before or during the implant surgery. Previous studies have demonstrated that the survival of implants placed in grafted areas could be even higher to those placed in pristine bone (Olson et al. 2000). In this sense, Franco et al. (2009) have reported less marginal bone loss for narrow implants placed in grafted areas, especially in the mandible, in comparison with those placed in non-grafted areas.

In summary, several studies evaluating the clinical outcome of narrow diameter implants placed in different indications are available. Narrow diameter implants supporting single tooth replacements have shown favourable clinical results (Mericske-Stern criteria of success. The International Journal of Oral 巴) Maxillofacial Implants 1: 11-25.

Allum, S.R., Tomlinson, R.A. \& Joshi, R. (2008) The impact of loads on standard diameter, small diameter and mini implants: a comparative laboratory study. Clinical Oral Implants Research 19: 553-559.

Andersen, E., Saxegaard, E., Knutsen, B.M. \& Haanaes, H.R. (2001) A prospective clinical study eval- et al. 2001; Zarone et al. 2006), even in the long-term outlook (Vigolo et al. 2004). Moreover, studies evaluating fixed partial dentures supported by narrow diameter implants have shown good clinical results, both after shortterm (Hallman 2001) and long-term follow-up periods (Comfort et al. 2005). Narrow diameter implants have also been used to support full arch reconstructions, and satisfactory results have been shown both for full arch fixed bridges (Zinsli et al. 2004), and overdentures in the mandible (Cho et al. 2007) and in the maxilla (Zinsli et al. 2004). The present study highlights the indications for narrow implants in compromised spaces, as an ideal therapeutic option to replace maxillary lateral incisors and mandibular incisors, in accordance with previous studies (Zarone et al. 2006). Nonetheless, longer follow-up periods are necessary to evaluate the actual behaviour of this therapeutic option.

\section{Conclusion}

OsseoSpeed $^{\mathrm{TM}}$ TX 3.0S implants are a safe and predictable treatment option in the anterior jaw region where physical space is limited. Mean marginal bone loss was stable up to 12 months after implant placement, and only $6.6 \%$ of the implants showed marginal bone loss of $1 \mathrm{~mm}$ or more. The surrounding soft tissue was stable and well maintained over time.

\section{Disclosure}

This international multicentre study has been fully sponsored by Astra Tech AB. However, none of the researchers have economical interests in the product related in this study or in the company.

\section{Acknowledgement: The authors} want to thank to Mr Robert Corbé for his help, guidance and monitoring process during this study. uating the safety and effectiveness of narrowdiameter threaded implants in the anterior region of the maxilla. The International Journal of Oral 4) Maxillofacial Implants 16: 217-224.

Anitua, E., Tapia, R., Luzuriaga, F. \& Orive, G. (2010) Influence of implant length, diameter, and geometry on stress distribution: a finite element analysis. International Journal of Periodontics and Restorative Dentistry 30: 89-95. 
Araujo, M.G., Sukekava, F., Wennstrom, J.L. \& Lindhe, J. (2005) Ridge alterations following implant placement in fresh extraction sockets: an experimental study in the dog. Journal of Clinical Periodontology 32: 645-652.

Arisan, V., Bolukbasi, N., Ersanli, S. \& Ozdemir, T. (2010) Evaluation of 316 narrow diameter implants followed for 5-10 years: a clinical and radiographic retrospective study. Clinical Oral Implants Research 21: 296-307.

Avila, G., Galindo, P., Rios, H. \& Wang, H.L. (2007) Immediate implant loading: current status from available literature. Implant Dentistry 16: 235 245.

Bengazi, F., Wennstrom, J.L. \& Lekholm, U. (1996) Recession of the soft tissue margin at oral implants. A 2-year longitudinal prospective study. Clinical Oral Implants Research 7: 303-310.

Block, M.S. \& Kent, J.N. (1993) Maxillary sinus grafting for totally and partially edentulous patients. Journal of the American Dental Association 124: 139-143.

Cardaropoli, G., Araujo, M. \& Lindhe, J. (2003) Dynamics of bone tissue formation in tooth extraction sites. An experimental study in dogs. Journal of Clinical Periodontology 30: 809-818.

Cardaropoli, G., Lekholm, U. \& Wennstrom, J.L. (2006) Tissue alterations at implant-supported single-tooth replacements: a 1-year prospective clinical study. Clinical Oral Implants Research 17: 165-171.

Cehreli, M., Akkocaoglu, M. \& Akca, K. (2006) Numerical simulation of in vivo intraosseous torsional failure of a hollow-screw oral implant. Head $\oplus$ Face Medicine 2: 36.

Chang, M., Wennstrom, J.L., Odman, P. \& Andersson, B. (1999) Implant supported single-tooth replacements compared to contralateral natural teeth. Crown and soft tissue dimensions. Clinical Oral Implants Research 10: 185-194.

Cho, S.C., Froum, S., Tai, C.H., Cho, Y.S., Elian, N. \& Tarnow, D.P. (2007) Immediate loading of narrow-diameter implants with overdentures in severely atrophic mandibles. Practical Proceedings in Aesthetic Dentistry 19: 167-174.

Comfort, M.B., Chu, F.C., Chai, J., Wat, P.Y. \& Chow, T.W. (2005) A 5-year prospective study on small diameter screw-shaped oral implants. Journal of Oral Rehabilitation 32: 341-345.

Cordaro, L., Torsello, F., Mirisola Di Torresanto, V. \& Rossini, C. (2006) Retrospective evaluation of mandibular incisor replacement with narrow neck implants. Clinical Oral Implants Research 17: 730-735.

Davarpanah, M., Martinez, H., Tecucianu, J.F., Celletti, R. \& Lazzara, R. (2000) Small-diameter implants: indications and contraindications. Journal of Esthetic Dentistry 12: 186-194.

Degidi, M., Piattelli, A. \& Carinci, F. (2008) Clinical outcome of narrow diameter implants: a retrospective study of 510 implants. Journal of Periodontology 79: 49-54.

Degidi, M., Piattelli, A., Iezzi, G. \& Carinci, F. (2007) Wide-diameter implants: analysis of clinical outcome of 304 fixtures. Journal of Periodontology 78: 52-58.

Franco, M., Viscioni, A., Rigo, L., Guidi, R., Zollino, I., Avantaggiato, A. \& Carinci, F. (2009) Clini- cal outcome of narrow diameter implants inserted into allografts. Journal of Applied Oral Sciences 17: 301-306.

Froum, S.J., Cho, S.C., Cho, Y.S., Elian, N. \& Tarnow, D. (2007) Narrow-diameter implants: a restorative option for limited interdental space. International Journal of Periodontics and Restorative Dentistry 27: 449-455.

Galindo-Moreno, P., Fauri, M., Avila-Ortiz, G., Fernandez-Barbero, J.E., Cabrera-Leon, A. \& SanchezFernandez, E. (2005) Influence of alcohol and tobacco habits on peri-implant marginal bone loss: a prospective study. Clinical Oral Implants Research 16: 579-586.

Hallman, M. (2001) A prospective study of treatment of severely resorbed maxillae with narrow nonsubmerged implants: results after 1 year of loading. The International Journal of Oral $\Theta$ Maxillofacial Implants 16: 731-736.

Jemt, T. (1999) Restoring the gingival contour by means of provisional resin crowns after singleimplant treatment. International Journal of Periodontics and Restorative Dentistry 19: 20-29.

Jofre, J., Hamada, T., Nishimura, M. \& Klattenhoff, C. (2010) The effect of maximum bite force on marginal bone loss of mini-implants supporting a mandibular overdenture: a randomized controlled trial. Clinical Oral Implants Research 21: 243-249.

Kanie, T., Nagata, M. \& Ban, S. (2004) Comparison of the mechanical properties of 2 prosthetic miniimplants. Implant Dentistry 13: 251-256.

Kinane, D.F. \& Lappin, D.F. (2002) Immune processes in periodontal disease: a review. Annals of Periodontology 7: 62-71.

Lee, Y.K., Kim, J.W., Baek, S.H., Kim, T.W. \& Chang, Y.I. (2010) Root and bone response to the proximity of a mini-implant under orthodontic loading. Angle Orthodontics 80: 452-458.

Lekholm, U. \& Zarb, G.A. (1985) Patient selection and preparation. In: Brånemark, P.I., Zarb, G.A. \& Albrektsson, T., eds. Tissue-Integrated Prostheses, 199-209. Chicago, IL: Quintessance Publishing Co. Loomer, P.M., Ellen, R.P. \& Tenenbaum, H.C. (1995) Characterization of inhibitory effects of suspected periodontopathogens on osteogenesis in vitro. Infection et Immunity 63: 3287-3296.

Martin, R.J. (2010) AstraTech OsseoSpeed 3.0 S Implant. Inside Dentistry April 2010: 2-4.

Matsushita, Y., Kitoh, M., Mizuta, K., Ikeda, H. \& Suetsugu, T. (1990) Two-dimensional FEM analysis of hydroxyapatite implants: diameter effects on stress distribution. Journal of Oral Implantology 16: 6-11.

Mericske-Stern, R., Grutter, L., Rosch, R. \& Mericske, E. (2001) Clinical evaluation and prosthetic complications of single tooth replacements by non-submerged implants. Clinical Oral Implants Research 12: 309-318.

Misch, C.E., Perel, M.L., Wang, H.L., Sammartino, G., Galindo-Moreno, P., Trisi, P., Steigmann, M., Rebaudi, A., Palti, A., Pikos, M.A., SchwartzArad, D., Choukroun, J., Gutierrez-Perez, J.L., Marenzi, G. \& Valavanis, D.K. (2008) Implant success, survival, and failure. The International Congress of Oral Implantologists (ICOI) Pisa Consensus Conference. Implant Dentistry 17: 5-15.

Ohkubo, C., Kobayashi, M., Suzuki, Y., Sato, J., Hosoi, T. \& Kurtz, K.S. (2006) Evaluation of tran- sitional implant stabilized overdentures: a case series report. Journal of Oral Rehabilitation 33: 416-422.

Olate, S., Lyrio, M.C., de Moraes, M., Mazzonetto, R. \& Moreira, R.W. (2010) Influence of diameter and length of implant on early dental implant failure. Journal of Oral \& Maxillofacial Surgery 68: 414-419.

Olson, J.W., Dent, C.D., Morris, H.F. \& Ochi, S. (2000) Long-term assessment (5 to 71 months) of endosseous dental implants placed in the augmented maxillary sinus. Annals of Periodontology 5: 152-156.

Petrie, C.S. \& Williams, J.L. (2005) Comparative evaluation of implant designs: influence of diameter, length, and taper on strains in the alveolar crest. A three-dimensional finite-element analysis. Clinical Oral Implants Research 16: 486-494.

Polizzi, G., Fabbro, S., Furri, M., Herrmann, I. \& Squarzoni, S. (1999) Clinical application of narrow Branemark System implants for single-tooth restorations. The International Journal of Oral $\Theta$ Maxillofacial Implants 14: 496-503.

Qian, L., Todo, M., Matsushita, Y. \& Koyano, K. (2009) Effects of implant diameter, insertion depth, and loading angle on stress/strain fields in implant/jawbone systems: finite element analysis. The International Journal of Oral $\oplus$ Maxillofacial Implants 24: 877-886.

Quek, C.E., Tan, K.B. \& Nicholls, J.I. (2006) Load fatigue performance of a single-tooth implant abutment system: effect of diameter. The International Journal of Oral @) Maxillofacial Implants 21: 929-936.

Reddy, M.S., O'Neal, S.J., Haigh, S., Aponte-Wesson, R. \& Geurs, N.C. (2008) Initial clinical efficacy of 3-mm implants immediately placed into function in conditions of limited spacing. The International Journal of Oral $\leftrightarrow$ Maxillofacial Implants 23: 281-288.

Renouard, F. \& Nisand, D. (2006) Impact of implant length and diameter on survival rates. Clinical Oral Implants Research 17(Suppl. 2): 35-51.

Romeo, E., Ghisolfi, M., Rozza, R., Chiapasco, M. \& Lops, D. (2006) Short (8-mm) dental implants in the rehabilitation of partial and complete edentulism: a 3- to 14-year longitudinal study. International Journal of Prosthodontics 19: 586592.

Saadoun, A.P. \& Le Gall, M.G. (1996) Implant site preparation with osteotomes: principles and clinical application. Practical Periodontics and Aesthetic Dentistry 8: 453-463.

Schwarz, M.S. (2000) Mechanical complications of dental implants. Clinical Oral Implants Research 11(Suppl. 1): 156-158.

van Steenberghe, D., Lekholm, U., Bolender, C., Folmer, T., Henry, P., Herrmann, I., Higuchi, K., Laney, W., Linden, U. \& Astrand, P. (1990) Applicability of osseointegrated oral implants in the rehabilitation of partial edentulism: a prospective multicenter study on 558 fixtures. The International Journal of Oral $\leftrightarrow$ ) Maxillofacial Implants 5: 272-281.

Tarnow, D.P., Cho, S.C. \& Wallace, S.S. (2000) The effect of inter-implant distance on the height of inter-implant bone crest. Journal of Periodontology 71: 546-549. 
Tarnow, D.P., Magner, A.W. \& Fletcher, P. (1992) The effect of the distance from the contact point to the crest of bone on the presence or absence of the interproximal dental papilla. Journal of Periodontology 63: 995-996.

Thilander, B., Odman, J. \& Jemt, T. (1999) Single implants in the upper incisor region and their relationship to the adjacent teeth. An 8-year follow-up study. Clinical Oral Implants Research 10: 346-355.

Vigolo, P. \& Givani, A. (2000) Clinical evaluation of single-tooth mini-implant restorations: a five- year retrospective study. Journal of Prosthetic Dentistry 84: 50-54.

Vigolo, P., Givani, A., Majzoub, Z. \& Cordioli, G. (2004) Clinical evaluation of small-diameter implants in single-tooth and multiple-implant restorations: a 7-year retrospective study. The International Journal of Oral ↔) Maxillofacial Implants 19: 703-709.

Winkler, S., Morris, H.F. \& Ochi, S. (2000) Implant survival to 36 months as related to length and diameter. Annals of Periodontology 5: 22-31.
Zarone, F., Sorrentino, R., Vaccaro, F. \& Russo, S. (2006) Prosthetic treatment of maxillary lateral incisor agenesis with osseointegrated implants: a 24-39-month prospective clinical study. Clinical Oral Implants Research 17: 94-101.

Zinsli, B., Sagesser, T., Mericske, E. \& MericskeStern, R. (2004) Clinical evaluation of smalldiameter ITI implants: a prospective study. The International Journal of Oral e) Maxillofacial Implants 19: 92-99. 